\title{
The Sufficientarian Alternative: A Commentary on Setting Health-Care Priorities
}

\author{
- Jay A. Zameska -
}

\begin{abstract}
In this commentary on Torbjörn Tännsjö's Setting Health-Care Priorities, I argue that sufficientarianism provides a valuable perspective in considering how to set health care priorities. I claim that pace Tännsjö, sufficientarianism does offer a distinct alternative to prioritarianism. To demonstrate this, I introduce sufficientarianism and distinguish two forms: Tännsjö's "weak sufficientarianism" and an alternative strong form of sufficientarianism that I call "revised lexical sufficientarianism." I raise a problem for Tännsjö's sufficientarianism, and advocate for the revised view on this basis. I then demonstrate that in the area of population ethics, the revised view outperforms the other views Tännsjö considers. As such, I aim to demonstrate that sufficientarianism - understood as its own theory and not just as a form of prioritarianism - offers unique advantages in population ethics, and would have been a valuable complement to the other theories Tännsjö considers.
\end{abstract}

Keywords: priority setting, health-care, sufficientarianism, distributive ethics, Torbjörn Tännsjö, Setting Health-Care Priorities, population ethics, repugnant conclusion, utilitarianism, maximin/leximin. Published online: 15 January 2020

\section{Introduction}

Torbjörn Tännsjö's Setting Health-Care Priorities provides a clear and insightful look at three major theories of distributive ethics - utilitarianism (with or without a prioritarian amendment), egalitarianism, and maximin/leximin - and draws out their theoretical and practical implications, ultimately considering how they may inform health systems. In this commentary, I argue that sufficientarianism provides a valuable perspective in considering how to set health care priorities. Sufficientarianism, the sufficiency view, or the principle of sufficiency, is the name for a family of related theories that hold that there is some threshold between good enough and bad lives, and that individuals' positions relative to this threshold are of special moral importance when assessing distributions. This view captured in slogan form - argues that what is most morally important is that people have 'enough,' understood as a sufficient amount of whatever currency is being distributed. Under Tännsjö's understanding, suffficientarianism is merely a form of prioritarianism. ${ }^{1}$

Jay A. Zameska

University of Tartu

Department of Philosophy

Jakobi 2

51005 Tartu, Estonia

Email: jay.allen.zameska@ut.ee

${ }^{1}$ Tännsjö (2019): 50. 
I argue that pace Tännsjö, sufficientarianism does offer a distinct alternative to prioritarianism, one that outperforms prioritarianism in population ethics. To demonstrate this, I introduce sufficientarianism and distinguish two forms: Tännsjö's "weak sufficientarianism" and an alternative, stronger "revised lexical sufficientarianism." I raise a problem for Tännsjö's sufficientarianism, and advocate a revised view on this basis. I then examine how the revised lexical view performs in population ethics compared to the other views Tännsjö discusses, arguing that sufficientarianism outperforms these other views. I support this by showing that sufficientarianism is not susceptible to Tännsjö's arguments for the repugnant conclusion. I end by suggesting that this shows strong, non-prioritarian forms of sufficientarianism to be plausible and attractive theories to consider when examining priority-setting in health care.

\section{Sufficientarianism: an overview}

The principle of sufficiency started with Harry Frankfurt in the mid-1980s. Frankfurt is credited with the canonical statement of sufficientarianism: "what is important from the point of view of morality is not that everyone should have the same but that each should have enough. If everyone had enough, it would be of no moral consequence whether some had more than others. ${ }^{2}$ This statement captures two important features of sufficientarianism, often called the positive thesis and the negative thesis. ${ }^{3}$ The positive thesis establishes the importance of people 'having enough.' This is usually conceptualized as reaching a particular morally privileged threshold of whatever 'currency' is being distributed. The positive thesis captures the core commitment of sufficientarian theories: that it is particularly important to benefit those below the threshold level. The negative thesis rejects the importance of other distributive requirements once people have enough.

Although the threshold is central to sufficientarianism, defining the threshold is an enduring challenge for sufficientarian theories. Establishing a precise threshold is often alleged to be impossible because any threshold seems to be either vague or arbitrary. ${ }^{4}$ However, the problem of vagueness is not unique to sufficientarianism: ${ }^{5,6}$ As such, I won't address how exactly to set the threshold here, though I will briefly discuss what motivates the threshold. Sufficientarianism is concerned with the distinction between good and bad lives (or more accurately, good enough and bad lives), and the threshold marks the boundary between the two. There are a variety of explanations of what makes a life "good enough" from a sufficientarian. ${ }^{7}$ In all of these wide-ranging cases, the core concern is the same: there is some threshold between good enough and bad lives, and individuals' positions relative to it are of special moral importance when assessing distributions.

\footnotetext{
${ }^{2}$ Frankfurt (1987): 21.

${ }^{3}$ Casal (2007).

${ }^{4}$ Fourie, Rid (2016): 2.

${ }^{5}$ Orr (2005): 22.

${ }^{6}$ Fourie, Rid (2016): 2.

${ }^{7}$ Cf. inter alia contentment (Frankfurt, 1987), utility levels related to important states (Benbaji, 2005), autonomy (Nielsen, 2016), the "conditions of freedom" (Shields, 2016).
} 
All sufficientarian views are committed to benefitting those below some morally-privileged level of well-being. However, they differ with how strongly they emphasize the importance of crossing the threshold. Strong views emphasize the importance of individuals crossing the threshold, while weak views emphasize benefiting those below the threshold without attaching any additional importance to crossing the threshold. In the remainder of this section, I present a brief overview of both strong and weak sufficiency views. This is not meant to be exhaustive, but only to highlight two important ends of the sufficientarian spectrum. Tännsjö espouses a weak view, which means that his view is a variant of prioritarianism. The strong view, in contrast, is not a form of prioritarianism. My primary claim is that this strong, non-prioritarian form of sufficientarianism should also be considered. To support this claim, first I attempt to lay out a plausible version of the strong form. Second, I demonstrate that both prioritarianism and weak sufficientarianism face serious problems that the strong view avoids. Then, I address a strong objection to strong sufficiency views: the excessive upwards transfers objection. I give reason to think that this objection is not fatal to the strong view as I present it. Following this, I examine the area of population ethics and demonstrate that the form of strong sufficientarianism I put forward here does not face the problems Tännsjö discusses (benign addition and the repugnant conclusion).

A common way to divide sufficientarian theories is between "weak" and "strong" views. ${ }^{8}$ The strength of a sufficiency view is typically a reference to how much importance is attached to ensuring individuals reach the threshold. For example, Harry Frankfurt's "headcount" sufficientarianism is a very strong view: its only concern is to maximize the number of people over the threshold, and so it attaches lexical priority to threshold-crossing benefits. ${ }^{9}$ On the other hand, there are also weaker sufficiency views which do not attach lexical priority to passing the threshold, but still strictly prioritize benefiting those below the threshold. Tännsjö proposes one such weak form of sufficientarianism. Tännsjö's sufficientarianism is a form of prioritarianism (a view that aims to maximize priority-weighted welfare) that includes "the idea that there is an upper limit [threshold] above which possible increases in happiness make no moral claim on us. We are allowed to make them, but we need not do so. And we are not allowed to make them if our doing so means any cost to individuals below the limit." ${ }^{10}$ In other words, Tännsjö's weak sufficiency holds that the goal of sufficiency is to maximize priority weighted welfare, with a priority function that ends at the threshold level. Tännsjö's view can be summarized as follows:

\footnotetext{
${ }^{8}$ See e.g. Segall (2016); Arneson (2002).

${ }^{9}$ Frankfurt (1987).

10 Tännsjö (2019): 50.
} 
Weak sufficientarianism: A state of affairs $x$, is better than a state of affairs $y$, if and only if:

1. There is greater priority-weighted welfare in $x$ than in $y$, where priority-weighted welfare has greater value the worse off its recipients are; or

2. There is at least as much priority-weighted welfare in $x$ as in $y$, and the amount of unweighted (i.e. above-threshold) welfare is greater in $\mathrm{x}$ than in $\mathrm{y}$.

Tännsjö's formulation is plausible and meets the 'core sufficientarian commitment.'11 However, as a variant of prioritarianism, it faces many of the same problems as prioritarianism, including (but not limited to) the repugnant conclusion. As such, weak sufficientarianism largely stands or falls in line with prioritarianism. Later, I'll raise a problem for both prioritarianism and weak sufficientarianism that strong sufficiency views can avoid.

As a contrast to Tännsjö's weak sufficiency view, I'll present a strong sufficiency view. The most famous strong view is Frankfurt's original "headcount" formulation of the principle of sufficiency, which aims to maximize the number of people above the threshold. His view, however, is typically considered to face a number of inescapably fatal problems. ${ }^{12}$ The headcount view represents one particular formulation of a lexical sufficiency view, because it places lexical priority on bringing people over the threshold. It is not the only possible lexical sufficiency view, however. As such, I propose what I call "revised lexical sufficientarianism" to take its place. ${ }^{13}$ Rather than aiming to maximize (priority-weighted) welfare, this revised view attempts to minimize the disvalue associated with below threshold lives. ${ }^{14}$ This revised view can be summarized as follows:

Revised lexical sufficientarianism: A state of affairs $x$ is better than a state of affairs $\mathrm{y}$ if and only if:

1. The number of people below the threshold is fewer in $x$ than in $y$; or

2. The number of people below the threshold is equal in $x$ and $y$, and the total shortfall from the threshold is less in $\mathrm{x}$ than in $\mathrm{y}$; or

3. The number of people below the threshold is equal in $x$ and $y$, and the total shortfall is equal in $\mathrm{x}$ and $\mathrm{y}$, and the total aggregate above-threshold welfare is higher in $x$ than $y$.

\footnotetext{
${ }^{11}$ Although not all would agree that weak sufficientarianism of this kind is distinct enough from prioritarianism to be considered a sufficientarian theory in its own right. See Segall (2016): 39.

${ }_{12}$ See e.g. Shields (2012); Casal (2007); Hirose (2016).

${ }^{13}$ Headcount sufficientarianism is a lexical view. It gives lexical priority to threshold-crossing benefits. My version is the "revised" lexical view (or revised lexical sufficientarianism), because it is an attempt to modify the headcount view while keeping its lexical priority on crossing the threshold. In this paper, when I say "lexical sufficientarianism" or the "revised view," I'm referring to the view I describe here, rather than to the headcount view or to strong/lexical sufficientarian views in general. ${ }^{14}$ Hirose (2016) proposes that a plausible axiological sufficiency view should stipulate that below-threshold lives have disvalue and aim to minimize this disvalue rather than to maximize welfare. The revised view I propose above follows Hirose's suggestion in this regard.
} 
Revised lexical sufficientarianism avoids the serious problems that afflict headcount sufficientarianism. First, in cases with a fixed population size, it is equivalent to headcount sufficientarianism, in that it aims to minimize the number of people below the threshold. ${ }^{15}$ However, in variable population size cases, it avoids the latter's implausible conclusions. Variable population size cases present a challenge for most theories, but they pose a special problem for headcount sufficientarianism. Consider the distributions $A(-5,-5,10)$ and $B(-5,-5,-5,-5,10)$ with a threshold at (10). Under the headcount view, $A$ is equally as good as B. But this is clearly implausible. In contrast, under the revised view, variable population size cases can be dealt with more satisfactorily, as a result of clause 1 . Per clause 1, adding more sub-threshold individuals always makes a state of affairs worse.

Second, it avoids the headcount view's complete insensitivity to depth. Headcount sufficientarianism cannot distinguish between cases where people are slightly below the threshold and cases where they are far below the threshold. Consider the following distribution: A $(-10,-10)$ and $B(-100,-1000)$. These distributions are equivalent under the headcount view. Under the revised view, B is clearly worse than A, thanks to clause (2) (in A, the total shortfall is 40 , in B, it is 1120). ${ }^{16}$

Additionally, these two problems pose a problem similar to the repugnant conclusion for headcount sufficientarianism that the revised view can avoid. ${ }^{17}$ Under a headcount view, $\left(11,-100_{1},-100_{2}, \ldots-100_{n}\right)$ is better than $(9,9,9)$, because in the former there is a higher incidence of sufficiency than the latter. I find this conclusion to be strongly counterintuitive. The revised lexical view does not face this problem, largely due to the theory's focus on minimization rather than maximization: there are more lives below the threshold in A than B, so A is worse. It also avoids a modified form of the problem where the number of people below the threshold is kept constant, but their position relative to the threshold is decreased. Per (2), revised lexical sufficientarianism holds that it is worse the farther below the threshold individuals are.

Third, headcount sufficientarianism cannot distinguish between distributions where everyone is sufficiently well off. For example, it cannot distinguish between

${ }^{15}$ The change from maximizing the incidence of sufficiency to minimizing the incidence of insufficiency maintains the spirit of the headcount view: it emphasizes the importance of bringing people over the threshold. It might be objected that this revised view is implausible because it may yield similar conclusions to negative utilitarianism - in particular, it faces what Casal (2007) calls the "empty world" objection. It holds that a world with no people is better than a world with any number of people just below the threshold. Some will undoubtedly find this to be objectionable. I don't find this objectionable at all - particularly when we consider that although maximizing theories avoid this objection, they entail the repugnant conclusion (discussed later). If I have to bite the bullet, I'll bite the bullet and happily prefer the empty world to the repugnant one.

${ }^{16}$ Note that, as it stands, the revised view is insensitive to below threshold distributions that have the same total shortfall. E.g. C $(-5,-15)$ is equivalent to A $(-10,-10)$ in this view. This lack of sensitivity is similar to total utilitarianism. However, some find this element implausible. Those who do may adjust my formulation to take account of priority-weighted shortfall from the threshold, where reducing a shortfall has greater value the further below the threshold the individual is. For my purposes here, it does not matter whether or not we adopted a weighted or unweighted view of the disvalue of shortfalls. In my discussion here, the primary relevant difference between weak sufficientarianism and revised lexical sufficientarianism is the level of priority attached to crossing the threshold.

${ }^{17}$ The repugnant conclusion is discussed later, in section 3. 
$A(11,11)$ and $B(1000,1000)$. In contrast, the revised view holds that $B$ is better because of clause 3. I don't have space to describe all of the differences between headcount sufficientarianism and revised lexical sufficientarianism, but I hope to have shown how the revised view avoids the largest problems of the headcount view, while still ascribing lexical priority to threshold-crossing benefits. As such, I take it to be a plausible reformulation of lexical sufficientarianism.

Although weak sufficiency and the revised lexical view share many similarities, these two sufficientarian views differ with regard to how they treat threshold-crossing benefits. The primary difference between the theories can be captured by:

Lexical priority: benefits that bring individuals past the threshold have lexical priority over benefits that do not. ${ }^{18}$

As a strong view, revised lexical sufficientarianism holds that threshold-crossing benefits have strict lexical priority over non-threshold-crossing benefits. In other words, a benefit that brings someone over the threshold is always worth more than a benefit that does not. ${ }^{19}$ The weak view is not bound to such a claim, because although it prioritizes benefitting those below the threshold, it does not place lexical priority on individuals reaching the threshold.

Strict lexical priority to threshold-crossing benefits is often considered to be a serious problem because it entails the upward transfers objection against strong sufficiency views. Often, weak sufficiency theories are preferred over their stronger counterparts on the basis of this objection..$^{20}$ In the next section, I argue that we need not think the upward transfers objection is a fatal problem, by demonstrating that the same features of revised lexical sufficientarianism that entail the upward transfers objection help it to avoid other problems that are at least as serious, problems that both weak sufficientarianism and prioritarianism face.

My aim has been to introduce the general notion of sufficientarianism and sketch out a couple important possibilities. In the next section, I argue that his view faces a problem, and suggest that we should accept lexical priority, which means accepting revised lexical sufficientarianism over weak sufficientarianism. The rejection of lexical priority is often motivated by avoiding a particular objection to strong sufficientarian views: the upward transfers objection. By countering this objection, I aim to defuse some of

\footnotetext{
${ }^{18}$ In this article, when I say "lexical priority" I'm referring specifically to this particular feature of strong sufficientarian views.

${ }^{19}$ This means that weak sufficientarianism is continuous, while revised lexical sufficientarianism is discontinuous. The revised view is discontinuous because it places lexical priority on minimizing the number of individuals below the threshold. This can be shown through a simple example. Let 10 be the threshold. Between distributions $X(9,9,18,18,18,18,18)$ and $Y(9,10,16,16,16,16,16), Y$ is better under the revised lexical view, even though 5 people stand to gain a larger benefit in $X$. This is because the value of moving from 9 to 10 outweighs the value of adding 2 to the other five people. As such, the revised view is strongly discontinuous. Notably, leximin would also yield the same judgement as the revised view, albeit for different reasons.

${ }_{20}$ And also sometimes to meet other theoretical commitments, such as continuity of value. The strong view requires value to be discontinuous, and some may find this implausible.
} 
the concern about lexical priority and undercut the motivation for preferring weak sufficientarian theories over their stronger counterparts.

\section{The upward transfers objection: a problem for lexical sufficientarian views}

In the previous section, I introduced revised lexical sufficientarianism. In this section I address the strongest objection against lexical sufficientarian views: the upward transfers objection. One of the main reasons why weaker sufficiency views are often preferred over stronger views is that they avoid the upward transfers objection (also called the "excessive upward transfers objection"). Richard Arneson has pressed a particularly strong form of this objection. ${ }^{21}$ Arneson argues that strong sufficiency views (like the one described above) are committed to providing the smallest possible threshold-crossing benefit to a single better off individual, over providing much larger benefits to a large number of worse off people. To use Arneson's own example:

Suppose millions of people are leaving lives of hellish quality, perhaps at the level of concentration camp victims. They can be raised to at best a moderate quality of life, close to the threshold. But there is some constraint that prevents us from enabling any of these hell residents from advancing to the threshold level. Still, we can bring about huge improvements in quality of life for huge numbers of people. We have one alternative choice: we could instead boost one individual whose prospects are currently just below the threshold level to prospects that are a tiny bit better and place her at the threshold. ${ }^{22}$

As Arneson points out, being committed to lexical priority requires us to prefer befitting one person by a small amount rather than many by a much larger amount. Arneson argues that this result is unacceptably counterintuitive. This is a particularly troubling objection, not only because it reveals seriously counterintuitive implications of the theory, but also because it seems at odds with the theory's commitment to helping the badly-off. However, I don't believe this objection is fatal to revised lexical sufficientarianism, and I hope to show that the revised view is at least as plausible as weak sufficiency and prioritarianism..$^{23} \mathrm{My}$ argumentative strategy is as follows. First, I aim to identify what component of the revised view yields the counterintuitive implications of the upward transfer objection. Then I determine what role this element plays in the theory, and assess whether or not the benefits are worth the cost (i.e. the upward transfer objection).

The central problem in the upward transfers objection is that apparently marginal benefits to one person can outweigh seemingly much larger benefits to many other people. This is a result of the revised lexical sufficientarianism's acceptance of lexical priority. Since the theory places lexical priority on reducing the number of lives below

${ }^{21}$ Arneson $(2002,2006)$.

${ }^{22}$ Arneson (2002): 188-189.

${ }^{23}$ Arneson thinks weaker sufficiency views are somewhat more plausible because they avoid the upward transfers problem, but he still finds them implausible overall due to issues with setting the threshold and with forgoing large above-threshold benefits. See Arneson $(2002,2006)$. 
the threshold, no amount of below threshold benefit can outweigh any amount of threshold-crossing benefit. As such, some apparently marginal benefit to a single person may outweigh some much larger sized benefits to many more people, so long as the former brings someone past the threshold and the latter do not.

Why should we accept lexical priority? At a broad level, I think such an approach helps to capture the core spirit of sufficientarianism, as espoused by Frankfurt's original formulation. However, more concretely - and more importantly - it helps us avoid conclusions that are at least as counterintuitive as Arneson's upward transfers objection. As an example, consider the following case from Yitzhak Benbaji, which he calls one pleasant day to the sick:

We can invest in a medicine that would enable one young person who suffers from a deadly disease to live a normal life. The other 99,999 equally young people who suffer from the same disease will die in two days. With the same amount of resources we can develop a medicine that will enable the whole group (100,000 people) one day of very pleasant life. Let us stipulate that this option yields more cross-personal aggregate utility than the utility gained by the medicine that completely cures only one person. ${ }^{24}$

Given the stipulation at the end, both prioritarianism and weak sufficientarianism are committed to providing the entire group with one pleasant day because it results in the largest aggregate utility gain. I find this to be deeply counterintuitive - at least as counterintuitive as Arneson's upward transfer objection. It seems far more valuable to provide someone with a full, decent life than provide any number of people with much less significant benefits ( $\mathrm{e}^{25} \mathrm{I}$ believe it is strong enough to present a serious problem for both weak sufficientarianism and prioritarianism.

However, if one pleasant day is not sufficiently counterintuitive, you may vary the length of time and the size of the population. For example, we might consider one pleasant minute, in which these young people gain a pleasant minute rather than a pleasant day. So long as the population of sick young people is sufficiently large, both prioritarianism and weak sufficientarianism must prefer distributing pleasant minutes over saving a life. ${ }^{26}$ In other words, there is some sufficiently large number of people that would obligate prioritarianism and weak sufficientarianism to prefer giving each of them one pleasant minute over giving someone a pleasant life. ${ }^{27}$

\footnotetext{
${ }^{24}$ Benbaji (2005): 335.

${ }^{25}$ Note that such an intuition need not be derived from sufficientarianism in particular. It seems to be the same intuition at work in Temkin's "Minimize great additional burdens" view (Temkin, 2005), and Caspar Hare's "Concentrate good effects" view (Hare, 2015: 133). Neither espouses a commitment to sufficientarianism, but they both share the intuition that there is something wrong with choosing to distribute pleasant days rather than save a life.

${ }^{26}$ If this is still not implausible enough, we could go even smaller: one pleasant second, one pleasant nano-second, and so on. As long as there is no limit on how large the population can be, we can create increasingly smaller pleasant moments that will outweigh a full life.

${ }^{27}$ Notably, this problem shares a similar structure with Arneson's objection. In both, some apparently marginal benefit (or benefits) outweigh(s) seemingly much larger benefit(s). In Arneson's objection, this is due to a lack of aggregation, while in Benbaji's case, it is the product of such aggregation.
} 
As stated above, I find one pleasant day to be strongly counterintuitive. Further, one pleasant day is not the only version of this problem that afflicts prioritarianism and weak sufficientarianism. ${ }^{28}$ Any number of similar problems can be constructed, so long as the theory allows for enough small benefits to many people to aggregate to outweigh a larger more significant benefit to a fewer number of people. Fortunately, the revised lexical view avoids one pleasant day and other similar problems. It does precisely because of lexical priority. In ascribing such priority, it essentially places a limit on what kinds of aggregation are permissible. No amount of above threshold welfare can aggregate to outweigh any decrease in the disvalue of below threshold welfare, and no amount of decrease in the disvalue of below threshold welfare can outweigh the decrease in the number of below threshold lives. This allows revised lexical sufficientarianism to avoid one pleasant day, and other related problems, but only at the expense of creating the upward transfers objection.

I think the upward transfers objection and one pleasant day are both deeply counterintuitive. I suspect that which is more counterintuitive for any given person will likely depend on that person's other theoretical commitments. However, I have more to say in preferring to avoid one pleasant day over the upward transfers objection. As I'll discuss in the next section, the same feature that allows the revised view to avoid one pleasant day (and that creates the upward transfer objection) also allows it to avoid the mere addition paradox and the repugnant conclusion. Overall, I suggest that since every theory yields counterintuitive conclusions in extreme cases (and I take both the upward transfer objection as phrased above, as well as one pleasant day, to be extreme cases), we'll have to accept counterintuitive conclusions at some point. As such, we might as well go with the theory that offers fewer of them - in this case, revised lexical sufficientarianism. In the next section, I demonstrate that in the area of population ethics, the revised view outperforms the other views Tännsjö considers.

\section{Population ethics: resisting the repugnant conclusion}

One of Tännsjö's main theoretical 'testing grounds' is population ethics. In this section, I examine Tännsjö's discussion of population ethics, focusing on two arguments he makes in favor of the repugnant conclusion. Tännsjö states the repugnant conclusion as "the conclusion that a world with ten billion extremely happy people (the A-world) is worse than a world (the Z-world) where many people (many enough) have lives barely worth living." ${ }^{29}$ Intuitively, it seems like the A world is better, and as such, a theory that claims the $\mathrm{Z}$ world is better appears to be flawed. Tännsjö, however, wants to challenge this conclusion. He offers two arguments for the repugnant conclusion and takes these arguments to apply to all three theories he considers. I'll challenge both, arguing that sufficientarianism can resist these two arguments, and thus, unlike the other theories he considers, does not have to accept the repugnant conclusion.

\footnotetext{
${ }^{28}$ See also, Chocolate to the well off (Benbaji, 2006), Misery for the ultra rich (Dorsey, 2013) as well as other similar aggregation problems like Lives for Headaches (Dorsey, 2009), and to some extent, Scanlon's Transmitter room (Scanlon, 2000: 235).

${ }^{29}$ Tännsjö (2019): 72.
} 


\subsection{Tännsjö’s first argument: not so repugnant?}

Tännsjö's first argument against the repugnance of the repugnant conclusion proposes that we do not really know how bad the $\mathrm{Z}$ world is. His aim is to demonstrate that preferring the $Z$ world is not as counterintuitive as it first appears. He argues that we likely have a "much too gloomy picture of the $\mathrm{Z}$ world," and in fact, the $\mathrm{Z}$ world may be full of lives very much like our own. ${ }^{30}$ His goal is not to demonstrate that the $\mathrm{Z}$ world is in fact better than the A world, but only to weaken our intuition that the $\mathrm{Z}$ world really is so bad. In Tännsjö's terms, he aims to demonstrate "there is something problematic with the intuition such that ... we cease to believe that it has any evidential value." ${ }^{31}$ In short, he argues that once we realize that the "value of the lives people live there [in the $Z$ world] may be similar to the value of the lives we live," we will no longer find the $Z$ world so repugnant. ${ }^{32}$

However, it is not clear that our lives truly are lives that are barely or only just worth living. Especially when thinking about the vast disparities in quality of life across the world, it seems like my life could be dramatically worse than it currently is, and yet still be very much worth living. This is further evidenced by the fact that people often suffer immense and terrible hardships and still affirm their lives as worth living, despite the suffering they endure. ${ }^{33}$ Presumably, if we were living lives barely worth living (like those in the $\mathrm{Z}$ world), significant decreases in well-being would make our lives not worth living. And yet, if what I've suggested above is true, even with significant losses in well-being, our lives remain worth living. As such, it seems unlikely to me that we are living lives like those in the $\mathrm{Z}$ world. Rather, it seems like we are living lives significantly better than those in the $Z$ world. If this is the case, then Tännsjö's argument is weaker than he presents it. The $\mathrm{Z}$ world may very well be as gloomy as we imagine. Consequently, it's unclear if Tännsjö's argument really does diminish the intuitive 'repugnance' of the repugnant conclusion.

The main upshot of the above is that if we want to determine just how repugnant the repugnant conclusion really is, we need some standard of comparison. This is where sufficientarian theories can play an important role. Sufficientarian theories feature a builtin standard of comparison: a privileged threshold of well-being that defines the border of a good enough life. This is most likely quite different from a life that is just barely worth living. ${ }^{34}$ We may speculate all we like as to how good or bad the $\mathrm{Z}$ world is, but with

\footnotetext{
30 Ibidem: 74.

${ }^{31}$ Ibidem: 73-73. Emphasis his.

32 Ibidem: 74.

${ }^{33}$ I should note that Tännsjö explicitly takes a hedonistic approach (Tännsjö, 2019: 9-11). As such, there is nothing in Tännsjö's view to suggest that we have any special insight into the overall balance of pleasure and pain in our life. However, it seems plausible to assume that individuals have at least as good (if not better) insight into the balance of pleasure and pain in their own lives as Tännsjö does. In other words, its not clear to me why we should accept Tännsjö's claim that our lives may be much like the lives of the $\mathrm{Z}$ world rather than individuals' own self assessments. Regardless, this element contributes further to the fact that it is unclear whether we live lives more like those in the A world or the $\mathrm{Z}$ world.

${ }^{34}$ While it is theoretically possible to set the sufficiency threshold at the level of 'life being worth living,' it would conflict with the primary normative motivation for sufficientarianism: that people should live good enough lives.
} 
a (fully specified) sufficientarian theory, we can actually know how good or bad it is. If many people in the $\mathrm{Z}$ world are below the threshold, then according to sufficientarianism it is a very bad world indeed. In a case like this, sufficientarianism gives us a clear preference for the A world. But if the people in the $\mathrm{Z}$ world are somehow actually above the threshold for decent lives, then we shouldn't find anything repugnant about it - there's nothing repugnant about a world where everyone is living a decent life, even if no one is living an excellent or extraordinary life. As such, it seems like sufficientarianism provides a benefit here that the other theories do not: it provides a built in way to check just how repugnant this conclusion is. And as I'll argue in the next section, sufficientarianism's threshold also prevents the theory from accepting Tännsjö's argument in favor of the repugnant conclusion.

\subsection{Tännsjö's second argument: benign addition}

In addition to trying to challenge our intuitions about the repugnance of the $\mathrm{Z}$ world, Tännsjö also provides an argument in favor of the repugnant conclusion. Tännsjö's argument starts from the A world, and involves continuously adding people at slightly lower levels of well-being than the currently existing people. Every time people are added, redistributions are performed to make them equal, ensuring that the worse-off always gain more than the better-off lose. In doing so, it is possible to move from the A world to the $Z$ world through merely adding value, because (according to Tännsjö) in each step those who are affected gain from our addition and redistribution. ${ }^{35}$ This argument isn't limited to utilitarianism either: he demonstrates that this move also works on both egalitarian and maximin/leximin theories. Tännsjö claims that even though these theories may not require the move to the $\mathrm{Z}$ world (as utilitarianism does) they do accept it, and have no theoretical capacities to block it. As such, he views this argument as "irresistible." 36

Looking more closely, this argument for the repugnant conclusion has a couple key components. First, according to Tännsjö, those who are affected gain from the move. This is what Tännsjö calls "the utilitarian intuition." ${ }^{37}$ This first aspect draws utilitarianism to the repugnant conclusion: each step increases total utility. Second, those who are added in each step are "grateful for being around." ${ }^{38}$ This second aspect is important for maximin/leximin and egalitarian theories. Key to Tännsjö's claim that both maximin/ leximin and egalitarian theories must accept these moves to the $\mathrm{Z}$ world is that the people added in each step "have no reasonable complaint to make against the fact that they've been added." ${ }^{39}$ However, both of these components of his argument for the repugnant conclusion are rejected by a sufficientarian approach.

First, sufficientarianism of the kind I describe above (in contrast to Tännsjö's version) does not primarily aim to maximize utility, and so is not bound to the first component of Tännsjö's argument. Instead, revised lexical sufficientarianism is primarily

\footnotetext{
35 Tännsjö (2019): 75.

${ }^{36}$ Ibidem: 80.

37 Ibidem: 75.

38 Ibidem.

39 Ibidem: 77.
} 
a theory of ,gap closures' - it prioritizes minimizing the number and depth of shortfalls from the sufficiency threshold over maximizing utility. As such, the mere fact that each of these moves increases utility has no independent significance for sufficientarianism. Rather, increases in utility only matter if these increases bring people closer to or over the threshold. Given that the $Z$ world is in all likelihood below the threshold a sufficientarian theory would identify, moves from the A world to the $\mathrm{Z}$ world are only acceptable insofar as they do not advance below the threshold. Thus, unlike utilitarianism, sufficientarianism does not require moving from the A world to the $\mathrm{Z}$ world.

Second, Tännsjö claims that under egalitarian and maximin/leximin approaches the people added in each step "have no reasonable complaint to make against the fact that they've been added." ${ }^{40}$ Consequently, when combined with the fact that each redistribution (1) improves the position of the worst-off and (2) ultimately decreases inequality, maximin/leximin and egalitarianism must accept these redistributions. This is not so for a sufficientarian theory. If people are introduced below the threshold, they do in fact have a reasonable complaint to make: sufficientarians believe that it is morally bad if someone is insufficiently well-off. In the same way that a utilitarian would object to adding people if this move somehow lowered total aggregate utility - claiming that it is in fact, wrong to do so - sufficientarians object when adding people increases instances of insufficiency. So, not only does sufficientarianism provide those added with a reasonable complaint to make - namely that they are now living insufficiently good lives - it also has absolutely no reason to perform these additions and redistributions, but significant reason not to. This is a dramatic contrast to the other theories Tännsjö considers, which all have some aspect of their theory that justifies these additions and redistributions.

So, if Tännsjö is right, proponents of egalitarianism and maximin/leximin may have no choice but to accept his argument for the repugnant conclusion. In contrast, sufficientarians do have a way to reject it: increasing instances of insufficiency is morally bad, and thus people added below the sufficiency threshold have a reasonable complaint to make. As a result, sufficientarianism permits us to follow the argument from benign addition down to the sufficiency threshold, but not below it.

In this section I've argued that sufficientarianism avoids Tännsjö's argument in favor of the repugnant conclusion. First, in contrast to utilitarianism, the headcount form of sufficientarianism I've identified is not a maximizing moral theory (in the sense that it denies that more utility is always better), and so does not require the creation of the repugnant $Z$ world. Second, in contrast to maximin/leximin and egalitarianism, sufficientarianism gives people grounds for reasonable complaint if they are added below the threshold and provides no justification for the below-threshold additions and redistributions. As such, this form of sufficientarianism also need not accept the argument for the repugnant conclusion.

I believe that this counts in favor of the theory, as it fits with widely held intuitions about the repugnant conclusion. However, it also demonstrates why sufficientarianism is a valuable theory to consider. As Tännsjö has explained, he is searching for

\footnotetext{
${ }^{40}$ Ibidem.
} 
distinct and plausible theories to inform how we should think about priority setting in health care ${ }^{41}$ My claim is that revised lexical sufficientarianism's performance in population ethics should give us a reason to consider sufficientarianism alongside the other three theories. ${ }^{42}$

\section{Conclusion}

In the preface, Tännsjö explains that he "cannot think of any important additional theory [he] should have discussed." ${ }^{33}$ Although it's impossible to discuss every theory, my hope is to have shown that sufficientarianism - understood as its own theory and not just as a form of prioritarianism - is a theory that is worthwhile to discuss. Although it is somewhat newer and less popular than the other theories considered in Setting HealthCare Priorities, sufficientarianism provides a distinctive point of view and offers plausible conclusions. It avoids problems faced by prioritarianism and weak sufficientarianism and outperforms both in population ethics, where the revised view provides a way to reject the repugnant conclusion.

\section{Bibliography}

Arneson R.J. (2002), “Why Justice Requires Transfers to Offset Income and Wealth Inequalities," Social Philosophy and Policy 19 (1): 172-200.

Arneson R.J. (2006), “Distributive Justice and Basic Capability Equality: 'Good Enough' is Not Good Enough," [in:] Capabilities Equality: Basic Issues and Problems, A. Kaufman (ed.), Routledge, New York-London: 17-43.

Benbaji Y. (2005), "The Doctrine of Sufficiency: A Defence," Utilitas 17 (3): 310-332.

Benbaji Y. (2006), "Sufficiency or Priority?," European Journal of Philosophy 14 (3): 327-348.

Casal P. (2007), “Why Sufficiency is Not Enough,” Ethics 117 (2): 296-326.

${ }^{41}$ Ibidem: ii-ix.

42 Additionally, although it is outside the scope of this article, sufficientarianism is likely to provide plausible practical guidance as well. Tännsjö claims that the theories under consideration result in an overlapping practical consensus when applied to priority setting in health care. The main practical upshot of this consensus is the need to redistribute resources from marginal life extension for the elderly to treating mental illness (Tännsjö, 2019: 174). Sufficientarianism is also likely part of this overlapping consensus. Tännsjö's primary focus - the question of marginal life extension for the elderly versus mental health care for the young - is likely a question of above- versus below-threshold benefits. The elderly seeking marginal life extension have presumably lived a sufficiently long life. Much like maximin/leximin, sufficientarianism provides a backward looking justification for denying marginal life extension: the elderly have lived sufficiently long lives, and are thus above the threshold. As such, they are not entitled to further life-extending care, although they are still entitled to palliative care to ensure moment-to-moment sufficiency (see: Davies, 2018) for discussion of these temporal aspects of sufficiency). So, although they must relinquish their claim on further life extension when others are insufficient, they are not abandoned. However, younger people afflicted with mental illness have clearly not lived lives of sufficient length and quality. They are, from this lifetime perspective, below the threshold. If they continue to live a normal lifespan in their current state, they will end up living a life that is insufficient. As such, they must take priority over marginal life extension for the elderly. As a result, it seems that when it comes to practical application, sufficientarianism is likely in line with the other theories Tännsjö discusses.

43 Tännsjö (2019): viii. 
Crisp R. (2003), “Equality, Priority, and Compassion,” Ethics 113 (4): 745-763.

Davies B. (2018), "Health (Care) and the Temporal Subject," Les ateliers de l'éthique/The Ethics Forum 13 (3): 38-64.

Dorsey D. (2009), "Headaches, Lives and Value," Utilitas 21 (1): 36-58.

Dorsey D. (2012), The Basic Minimum: A Welfarist Approach, Cambridge University Press, Cambridge.

Dorsey D. (2013), “Equality-Tempered Prioritarianism," Politics, Philosophy \& Economics 13 (1): 45-61.

Fourie C., Rid A. (2016), "Introduction," [in:] What is Enough?: Sufficiency, Justice, and Health, C. Fourie, A. Rid (eds.), Oxford University Press, Oxford: 1-8.

Frankfurt H. (1987), “Equality as a Moral Ideal,” Ethics 98 (1): 21-43.

Hare C. (2015), "Statistical People and Counterfactual Indeterminacy," [in:] Identified Versus Statistical Lives: An Interdisciplinary Perspective, I.G. Cohen, N. Daniels, N. Eyal (eds.) Oxford University Press, New York: 124-135.

Hirose I. (2016), “Axiological Sufficientarianism," [in:] What is Enough?: Sufficiency, Justice, and Health, C. Fourie, A. Rid (eds.), Oxford University Press, Oxford: 51-68.

Nielsen L. (2015), "Sufficiency Grounded as Sufficiently Free: A Reply to Shlomi Segall," Journal of Applied Philosophy 33 (2): 202-216.

Orr S.W. (2005), "Sufficiency of Resources and Political Morality," paper delivered to the Priority in Practice seminar, 22 September 2005, University College London.

Scanlon T. (2000), What We Owe to Each Other, Belknap Press, Cambridge (MA).

Segall S. (2016), “What is the Point of Sufficiency?," Journal of Applied Philosophy 33 (1): 36-52.

Shields L. (2012), “The Prospects for Sufficientarianism," Utilitas 24 (1): 101-117.

Shields L. (2016), Just Enough: Sufficiency as a Demand of Justice, Edinburgh University Press, Edinburgh.

Temkin L.S. (2003), “Equality, Priority or What?," Economics and Philosophy 19 (1): 61-87.

Temkin L.S. (2005), “A ‘New' Principle of Aggregation," Philosophical Issues 15 (1): 218-234.

Tännsjö T. (2019), Setting Health-Care Priorities: What the Ethical Theories Tell Us, Oxford University Press, Oxford. 